\title{
Phase diversity restoration of sunspot images
}

\section{Dynamics around a decaying sunspot}

\author{
J. A. Bonet ${ }^{1}$, I. Márquez ${ }^{1,4}$, R. Muller ${ }^{2}$, M. Sobotka ${ }^{3}$, and Th. Roudier ${ }^{2}$ \\ 1 Instituto de Astrofísica de Canarias, 38205 La Laguna, Tenerife, Spain \\ e-mail: jab@ll.iac.es \\ 2 Observatoire du Pic du Midi, 57 avenue d'Azereix, BP 826, 65008 Tarbes, France \\ 3 Astronomical Institute, Academy of Sciences of the Czech Republic, 25165 Ondřejov, Czech Republic \\ ${ }^{4}$ Departamento de Análisis Matemático de la Universidad de La Laguna, 38271 La Laguna, Tenerife, Spain
}

Received 26 May 2004 / Accepted 20 September 2004

\begin{abstract}
Two time series, taken simultaneously in the $G$-band and in white-light, and corrected for telescope aberrations and turbulence perturbations using the method of phase diversity, are employed to study the motions of granules and $G$-band bright points (GBPs) in the moat of an old regular sunspot. Local correlation tracking and feature tracking have been utilized for this purpose. A large-scale radial outflow with a mean velocity of $0.51 \mathrm{~km} \mathrm{~s}^{-1}$ has been measured in the sunspot moat. Centres of diverging horizontal motions, identified with families of granules formed by repeatedly splitting granules, move away from the sunspot. Most of the GBPs in the moat also move outwards through radially orientated "channels" (confined between the borders of adjacent families) with velocities comparable to those of the adjacent granules. However, $6 \%$ of the GBPs move faster $\left(>1.4 \mathrm{~km} \mathrm{~s}^{-1}\right)$ than the neighbouring granules. GBPs in the moat are not regularly distributed but they are less frequent on its solar centre side.
\end{abstract}

Key words. Sun: photosphere - Sun: sunspots - methods: data analysis - Sun: granulation - Sun: magnetic fields techniques: image processing

\section{Introduction}

In the first paper of this series (Bonet et al. 2004; hereafter Paper I), we investigated the relation between penumbral and photospheric features in a decaying sunspot in order to better understand how the convection interacts with the sunspot magnetic field. The main results of Paper I can be summarized as follows: some outward moving penumbral grains cross the penumbra-photosphere boundary and continue moving as small bright features (as bright as regular granules, but not as bright as $G$-band bright points in $G$-band filtergrams), or they expand and develop into structures of the size and brightness of granules; $G$-band bright points (GBPs) are not born inside sunspot penumbra, but outside, often close to dark penumbral fibrils just at the penumbra-photosphere boundary.

The latter result confirms the finding by Ryutova et al. (1998) that the magnetic features moving away from sunspots frequently appear along the continuation of dark filaments. The magnetic flux is then carried away to the surrounding network throughout the moat (Sheeley 1969; Vrabec 1971; Harvey \& Harvey 1973; Wallenhorst \& Topka 1982; Muller \& Mena 1987; Ryutova et al. 1998) which is an annular cell of systematic horizontal outflows, 0.5 to $1 \mathrm{~km} \mathrm{~s}^{-1}$, surrounding sunspots in late phases of evolution (Sheeley \& Bhatnagar 1971;
Sheeley 1972; Muller \& Ména 1987). Soon after the discovery of this flow, which is very similar to that found in supergranules, Sheeley (1972) suggested that a decaying sunspot occupies the centre of a supergranular cell, and that small-scale fragments of magnetic flux are carried away from sunspots by the supergranular flows. This was observed, among others, by Harvey \& Harvey (1973) in a series of magnetograms, and by Muller \& Ména (1987) in a series of high-resolution images taken in the $G$-band (around $4305 \AA$ ), showing many local brightenings, the GBPs, moving away from the sunspot. Schmidt et al. (1985) suggested that this process contributes to the decay of sunspots.

As in supergranules in the quiet Sun, Muller \& Ména (1987) and Shine et al. (1987) found that the granules in the moat are advected towards its outer boundary. In addition to this systematic drift, granules exhibit local horizontal motions, mostly related to splitting and expansion. In the quiet photosphere far from sunspots, repeatedly splitting granules produce long-lived structures called "families of granules" (Kawaguchi 1980; Roudier et al. 2003). Using a feature-tracking technique, the latter authors found that a significant fraction of granules are organized in the form of spatio-temporal "trees of fragmenting granules," equivalent to families of repeatedly 
splitting granules, originating from a single granule at the beginning. Families with lifetimes longer than 1.5 h cover $62 \%$ of the total area. Such long-lived families produce coherent diverging flows that can be identified to mesogranules. The cumulative effect of a sequence of different less long-lived families partially overlapping in time can also produce diverging flow (Roudier \& Muller 2004).

Muller \& Ména (1987) found from measurements in the sunspot moat that GBPs move more rapidly on average than the granules. If true, the difference of velocities would show that the magnetic field does not move passively, being carried away by the convective flow, but rather that the magnetic field and convective flows interact in a complex way (Schmidt et al. 1985; Ryutova et al. 1998; Thomas et al. 2002). Making use of the excellent quality and continuity of our observing material, one of the aims of this paper is to compare the motion and the velocity of granules and GBPs in the moat. Since we are mainly interested in concluding whether the convective structures and GBPs are transported by the same large scale steady outflow, we consider the granules in our segmentation as global objects regardless of their internal structure. "Local correlation tracking" and "feature tracking" techniques are commonly used to follow the motion of structures on the solar surface. We will employ the same tracking method for both structures to prevent a possible differential bias between both techniques. Feature tracking is the more suitable tool for this purpose since the motion of GBPs can better be tracked this way. The relative motion must be studied very locally for adjacent GBPs and granules to ensure that they are both carried by the same flow.

The present paper is an extension of Paper I based on two 2-h series of sunspot images reconstructed with the phase diversity technique (henceforth PD). The long duration of the series, together with the excellent and stable quality during the whole period, makes the identification and tracking of small features possible (see Fig. 1 of this paper and also movies at the web site: http://www.iac.es/proyect/solarhr). We concentrate on horizontal motions in the surroundings of a decaying sunspot. The velocity fields in granulation around the sunspot are analysed. Also, the relation between the families of granules and the centres of diverging flows in the sunspot moat is studied. Finally, the velocities of GBPs and adjacent granules are re-compared using feature-tracking techniques for both types of objects.

\section{Observations and data processing}

Two simultaneous time series of images in white-light $(\lambda 450.7 \mathrm{~nm}, F W H M=0.9 \mathrm{~nm})$ and in the $G$-band $(\lambda 430.8 \mathrm{~nm}$, $F W H M=1.1 \mathrm{~nm})$, respectively, were obtained on 1999 July 7 at the Swedish Vacuum Solar Telescope (SVST, Observatorio del Roque de los Muchachos, La Palma - see Scharmer et al. 1985). The series follow the evolution of the leading sunspot of a decaying bipolar group NOAA 8620, close to the solar disc centre at $\mu=0.93$, for more than two hours.

The observational strategy for each observing channel consisted in taking pairs of simultaneous focused-defocused images in order to apply later the PD technique for image reconstruction (Gonsalves \& Childlaw 1979; Gonsalves 1982;

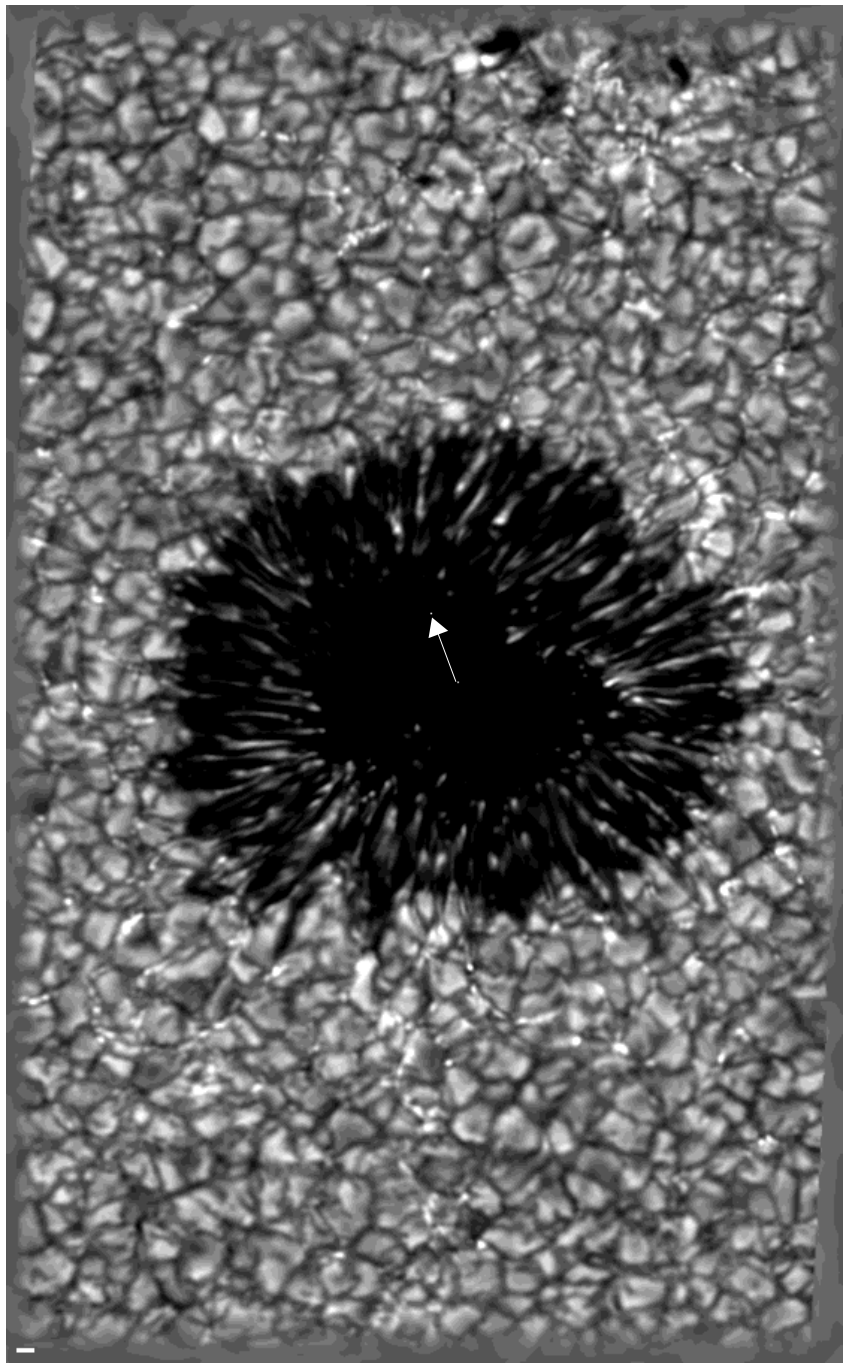

Fig. 1. A $G$-band image of the field studied (08:04 UT), restored with the phase diversity technique. The darkest levels of the grey scale at the sunspot centre are saturated. A set of small pores lies at the upper right corner of the field. The white bar in the lower left corner is $1^{\prime \prime}$ long and 0.'25 thick. Gray diffuse borders of the frame stem from the apodization in the subsonic filtering process. The arrow in the centre of the sunspot points to the solar disc centre.

Paxman et al. 1992; Löfdahl \& Scharmer 1994;Paxman et al. 1996). This technique allows a subtantial correction for the aberrations induced by both the telescope and the turbulent terrestrial atmosphere.

Prior to restoration, all images were pre-treated for darkand flat-field corrections. The restored images were derotated, aligned, destretched, and filtered for $p$-modes in $k-\omega$ space (threshold phase velocity $=5 \mathrm{~km} \mathrm{~s}^{-1}$ ). Two movies were produced, for white-light and the $G$-band, of 288 frames each, spanning over $2 \mathrm{~h}(\Delta t=25 \mathrm{~s})$ and covering a field of view (FOV) of $46^{\prime \prime} \times 75^{\prime \prime}(560 \times 910$ pixels $)$, with the sunspot located in the centre.

Apart from the PD observations at the two wavelength bands mentioned above, a complementary data set was acquired in the Ca II K line $(\lambda 393.3 \mathrm{~nm}, F W H M=0.3 \mathrm{~nm})$ at a mean rate of one image per $30 \mathrm{~s}$ interval, for monitoring the magnetic activity in the observed field. Figure 2 a shows a 

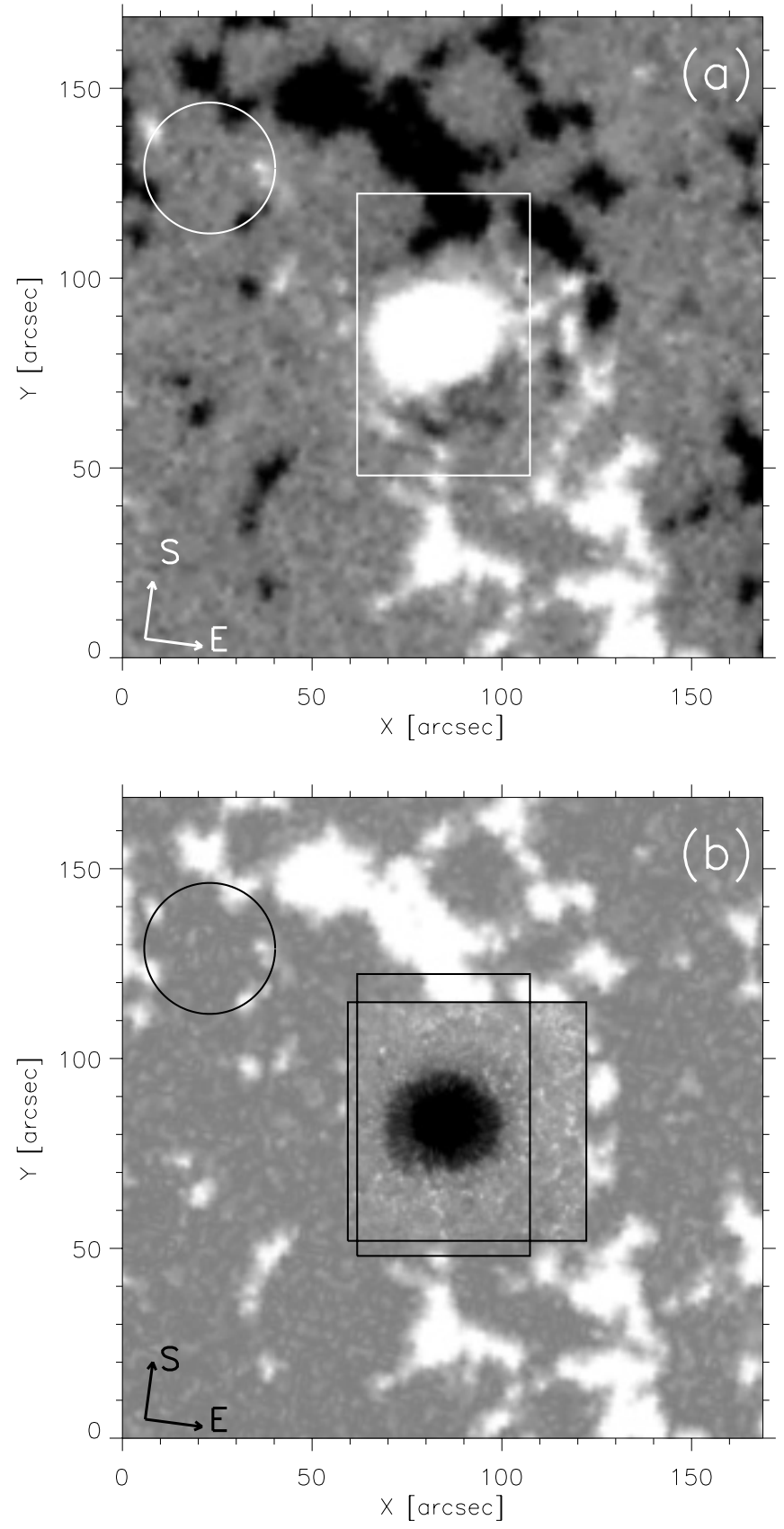

Fig. 2. a) Magnetogram from part of the SOHO/MDI full-disk magnetogram (at 08:00 UT) covering a wide region around the field studied. The signal is saturated at $\pm 100 \mathrm{G}$. b) Ca II K image taken at 07:57 UT (confined by the black square) overlying the magnetic signal in absolute value. The area within the rectangles in panels a) and b) corresponds to the field studied in the white-light and $G$-band time series throughout this article. The circles in the upper right corners represent a reference size for supergranulation cells $\left(\sim 35^{\prime \prime}\right)$.

magnetogram from SOHO/MDI (Scherrer et al. 1995) in a wide region around the area of interest. The MDI signal in the quietest areas of our FOV is $(1 . \pm 7.4) \mathrm{G}$, with the rms matching the MDI noise (e.g. $6.9 \mathrm{G}$ for Hagenaar 2001). In Fig. 2 b polarimetric signals in absolute value are represented and overlying is a Ca II $\mathrm{K}$ image (confined by the black square), taken at approximately the same time, that has been employed to precisely
Table 1. Rough average values of magnetic flux density in different regions of the FOV studied in white-light and the $G$-band (extracted from the SOHO/MDI magnetogram at 08:00 UT).

\begin{tabular}{lc}
\hline \hline Region in the FOV & $\begin{array}{c}\text { Mag. signal [G] } \\
\text { (absolute value) }\end{array}$ \\
\hline Umbra & 1370. \\
Penumbra (solar limb side) & 540. \\
(solar centre side) & 400. \\
$\quad$ (total average) & 450. \\
Moat (solar limb side) & 20. \\
(solar centre side) & 23. \\
(total average) & 30. \\
Supergranular boundary & 60. \\
Quiet areas & 6. \\
Active area above the sunspot & $250-530$. \\
\hline
\end{tabular}

locate the position of the observed field in the magnetogram. The area within rectangles in panels (a) and (b) corresponds to the FOV studied in the white-light and $G$-band time series throughout this paper. The borders of two supergranular cells intersect this FOV at the lower and upper left corners, respectively. The trace of these borders is also present in the map of mean horizontal velocities shown in Fig. 4. Apart from in the sunspot, the magnetic signal is also enhanced in the upper central and right parts of the FOV, i.e. in the region of the small pores. Table 1 summarizes rough average values of the unsigned magnetic signal in different regions of the FOV. On the solar limb side of the penumbra the magnetic signal is enhanced as compared to the opposite side. In the moat no significant difference between either side is measured although a remarkable enhancement of the signal is found in its upper right $\operatorname{part}(57 \mathrm{G})$.

A glance at Fig. 2a reveals the nearly circular moat bounded by magnetic structures.

To isolate GBPs, the white-light images were aligned exactly with the corresponding $G$-band frames and their granular contrast was reduced to match the contrast observed in the $G$-band. The white-light frames were then subtracted from the $G$-band ones, thus producing intensity difference images with the GBPs substantially enhanced. The segmentation was done by thresholding and multiplying the obtained binary masks by the original $G$-band frames.

The segmentation of granulation was performed in the white-light images following the method by Strous (1994). For further details of the observations, image treatment and segmentation techniques we refer the reader to the Paper I of this series.

\section{Results}

\subsection{Motions of granules in the surroundings of the sunspot}

The white-light images were used to study horizontal motions of granules in the full $46^{\prime \prime} \times 75^{\prime \prime}$ FOV by means of a local 

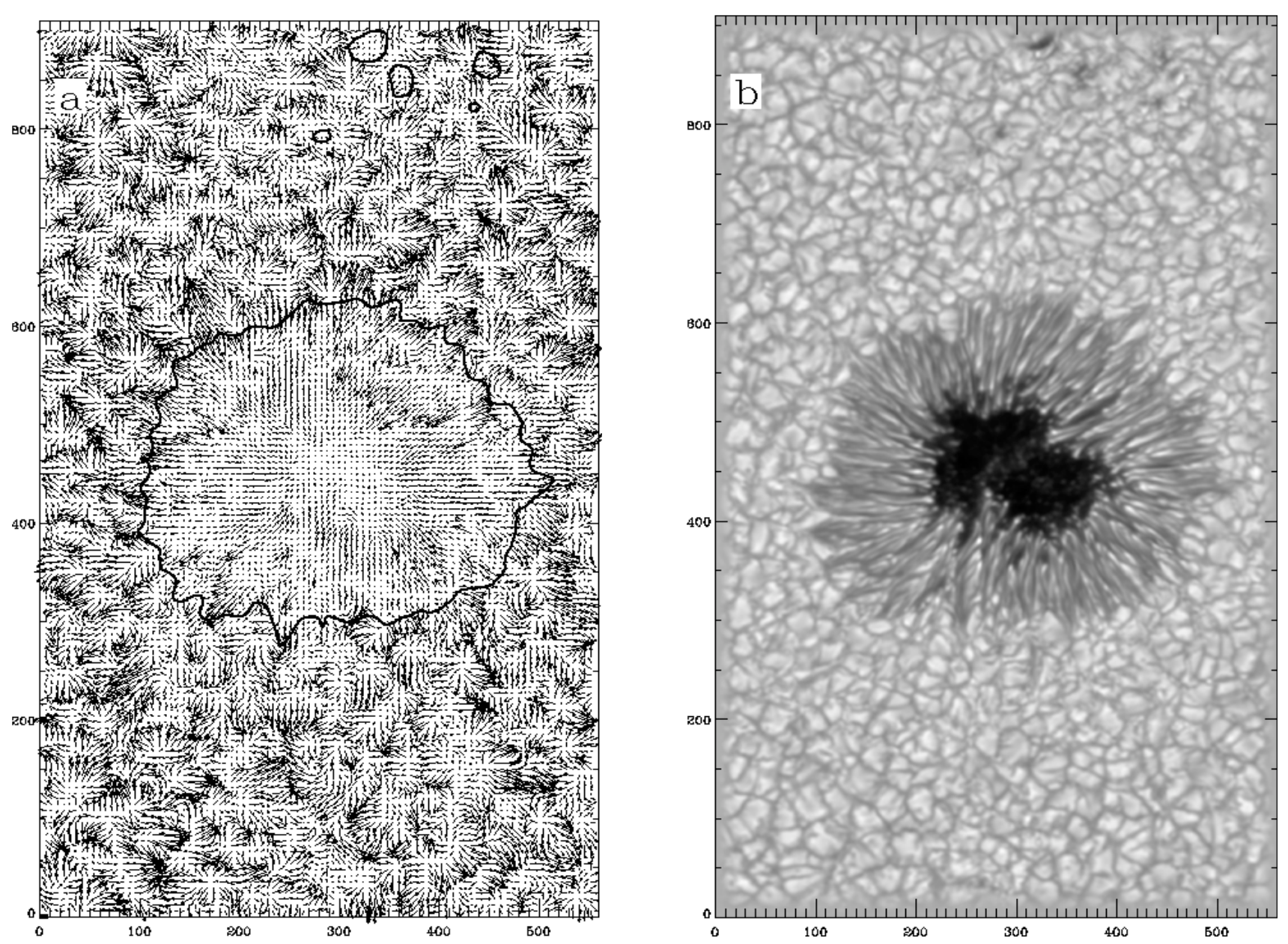

Fig. 3. a) One of the 24 maps of horizontal velocities, averaged over $5 \mathrm{~min}$. The coordinate unit is 0. '083 (1 pixel). The length of the black bar at coordinates $(0,0)$ corresponds to $1 \mathrm{~km} \mathrm{~s}^{-1}$. b) Average image of the correponding 5-min white-light series.

correlation tracking method (LCT, November \& Simon 1988; computer code by Molowny-Horas \& Yi 1994). The FWHM of the Gaussian tracking window was set to 0!'75 (approximately the half of the typical granular size) and the time-averaging interval to $5 \min$ (12 frames). A sequence of 24 velocity maps was obtained during the 2-h period of the time series. One of the maps (the thirteenth in the sequence) is shown in Fig. 3a. Numerous centres of diverging horizontal motions, of average size of $3^{\prime \prime}$, caused mostly by expansion and splitting of granules and commonly associated with mesogranules (Roudier et al. 2003; Roudier \& Muller 2004), are present in the whole field outside the sunspot. In this paper we call them "rosettas", a term that has been used by several authors like Sobotka et al. (1999) or Roudier et al. (2002). For the sake of clarity, we will define the meaning that we assign to the terms rosetta and mesogranule. Rosetta refers to divergences in flow vector maps computed with high resolution integration parametres (typically 0.7 and 5-10 min); they correspond to "strong positive divergences among which the exploding granules are the most energetic" (Rieutord et al. 2000). This is the kind of divergence structures shown in Fig. 3a. Mesogranules correspond to complexes of granules exploding and expanding recurrently and they are detected with larger integration parametres (typically $1 . " 5-5$ ". and $15-30 \mathrm{~min}$ ); they are related to the families of granules as we will discuss below. For comparison with the velocity fields in Fig. 3a, an average image calculated for the corresponding 5-min white-light time series is shown in Fig. $3 b$.

A movie composed of these 24 velocity maps shows that in the moat surrounding the sunspot the rosettas move away from the spot. To study this motion, we converted the velocity maps into a series of images of divergence structures, i.e. images mapping the values of the divergence in a grey scale representation. Since these structures change their shape strongly with time, we had to remove this disturbing small-scale effect by smoothing the images with a Gaussian kernel of $F W H M=$ 1."5, half the typical size of the rosettas. Thus, only significant tracers, representing positions of the rosettas, were preserved. The LCT algorithm, with the FWHM of the Gaussian tracking window of $3^{\prime \prime}$ and the temporal averaging interval of $2 \mathrm{~h}$, was then applied to the series of 24 smoothed images of divergence. The resulting velocity map is shown in Fig. 4. The moat, $8^{\prime \prime}$ wide on average, can be seen quite clearly in the figure. For the following analysis, the moat region is defined empirically as an area between the sunspot border and the isovelocity line of $0.32 \mathrm{~km} \mathrm{~s}^{-1}$. This line was found to be the best separation between the regular motions directed from the spot and the other motions in the field. 


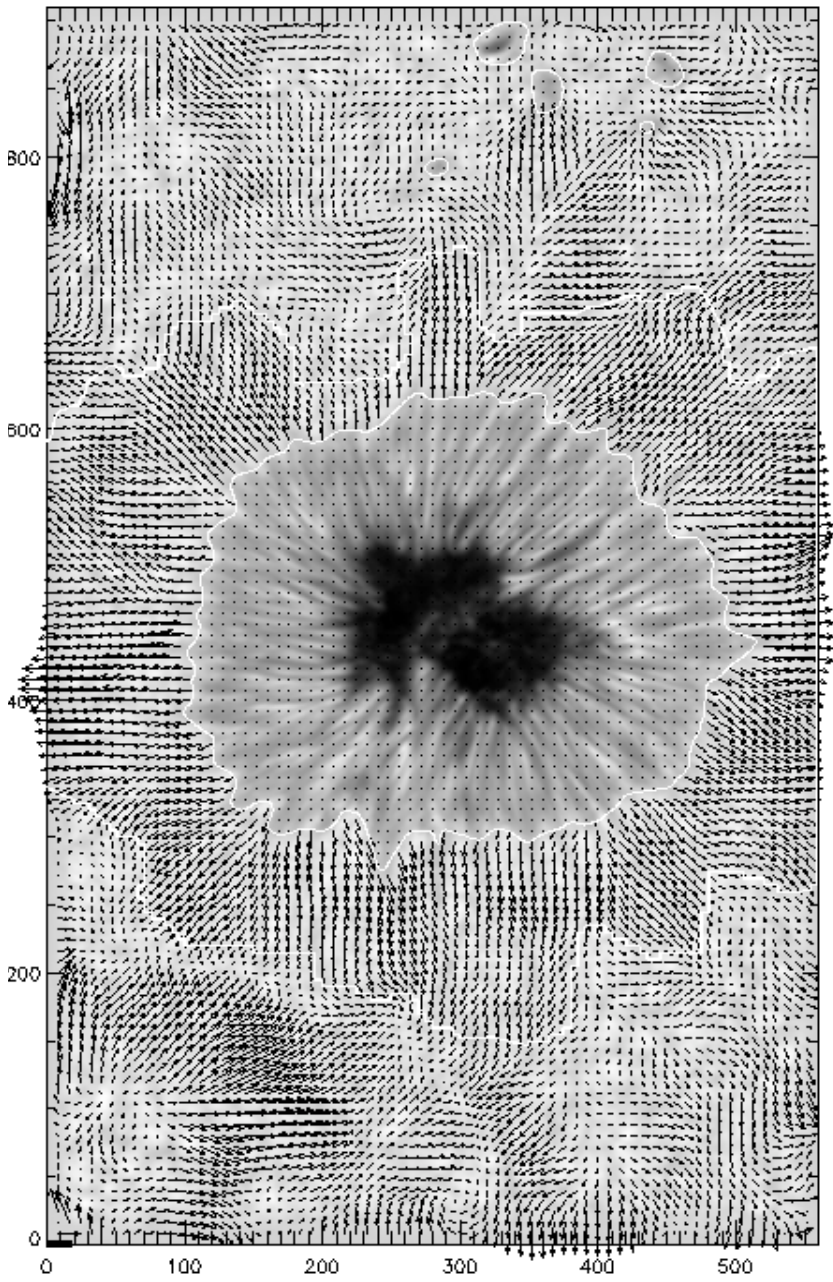

Fig. 4. Map of horizontal velocities of divergence structures, showing large-scale flows. The coordinate unit is $0{ }^{\prime} 083$ ( 1 pixel). The length of the black bar at coordinates $(0,0)$ corresponds to $1 \mathrm{~km} \mathrm{~s}^{-1}$. Note that the velocity scaling differs from Fig. 1 . The velocities inside the spot were set to zero.The background represents the average image of the white-light series. The moat region is outlined by a white contour.

According to the size of the tracking windows and integration times fixed in the previous paragraphs it can be concluded that Fig. 4 gives essentially account of the large-scale steady or quasi-steady velocity flow transporting the divergence centers $\left(\boldsymbol{v}_{\mathrm{d}}\right)$, whereas Fig. 3a represents granular velocities $\left(\boldsymbol{v}_{\mathrm{g}}\right)$ resulting from the combination in a vector summation of two components, namely the expansion and splitting of granules associated with mesogranular flows $\left(\boldsymbol{v}_{\mathrm{g}}^{\prime}\right)$ and the large-scale flow driving the divergence centers. Accordingly, the "net" expansion velocities in rosettas can be obtained, at least to a first approximation, as: $\boldsymbol{v}_{\mathrm{g}}^{\prime}=\boldsymbol{v}_{\mathrm{g}}-\boldsymbol{v}_{\mathrm{d}}$.

Figure 4 shows in the sunspot moat the large-scale regular outflow that carries granules and divergence centres away from the sunspot. We can also observe in Fig. 4 that the velocities of the divergence structures are generally smaller at the top of the field of view (above the moat), where several small pores are present, compared to the bottom part (below the moat), where speeds of up to $1 \mathrm{~km} \mathrm{~s}^{-1}$ are observed in the coordinate range (0-250, 0-200). These high velocities are related to a part of
Table 2. Mean velocities in different regions $\left(\mathrm{km} \mathrm{s}^{-1}\right)$. Standard deviations characterize the width of the distribution of measured speeds.

\begin{tabular}{lccc}
\hline \hline Region & Moat & Bottom & Top \\
\hline Granules & & & \\
(5 min avg.) & $0.79 \pm 0.43$ & $0.80 \pm 0.44$ & $0.73 \pm 0.41$ \\
Large-scale flows & $0.51 \pm 0.13$ & $0.36 \pm 0.21$ & $0.25 \pm 0.12$ \\
Rosetta expansion & $0.64 \pm 0.36$ & $0.72 \pm 0.38$ & $0.68 \pm 0.39$ \\
Centroids of & & & \\
$\quad$ families & $0.54 \pm 0.21$ & $0.47 \pm 0.23$ & $0.34 \pm 0.18$ \\
\hline
\end{tabular}

the large regular supergranule adjacent to the moat, observed in the lower left corner of our FOV (see Fig. 2).

Mean values of velocity magnitudes, together with standard deviations that characterize the dispersion of individual speeds, are summarized in Table 2 for the moat and for the bottom (below the moat) and top (above the moat) parts of the FOV. The first row in the table corresponds to $\boldsymbol{v}_{\mathrm{g}}$, as derived from the 24 velocity maps averaged over 5 min (like that in Fig. 3a). The mean velocities of granules are nearly equal in the moat and in the bottom part of the field, which contains quiet granulation and a supergranular boundary. The top region with granulation and small pores shows a reduced mean velocity due to the presence of high magnetic signal in and around the pores. The second row in the table describes the mean behaviour of $\boldsymbol{v}_{\mathrm{d}}$ (Fig. 4). The mean velocity of divergence structures is largest in the moat, where the velocities are organized in a radial pattern, and smallest in the top region with pores. The values in the third row represent the mean expansion velocities in the rosettas: $\boldsymbol{v}_{\mathrm{g}}-\boldsymbol{v}_{\mathrm{d}}$. These velocities are largest in the bottom region, where the magnetic field is weak, and lowest in the moat. This indicates that the magnetic field dispersed in the moat and around the pores reduces the expansion speeds in the granulation.

\subsection{Families of granules in the surroundings of the sunspot}

An alternative description of motions and evolution of granules can be made in terms of families of granules. To do this, each white-light frame is segmented, i.e. converted into a mask where pixels belonging to granules have non-zero values and the rest are set to zero. The evolution of granules is then tracked in time, taking appearances, disappearances, splitting and merging into account. A granule during its lifetime and all granules originating from it by splitting are labelled by a unique number. If some of these granules split again, more and more granules are labelled by the same number and form a family. This technique is described in detail by Roudier et al. (2003).

In total, 269 families living longer than $1 \mathrm{~h}$ were detected in the field of view: 131 in the moat (defined in Sect. 3.1), 70 in the part below the moat, and 68 in the pore region above the moat. The presence of long-lived families in the moat indicates that the creation of new granules by splitting is usual also near the sunspot. Each family, at a given time, is characterized by its area and by the position of the centroid of this area. 


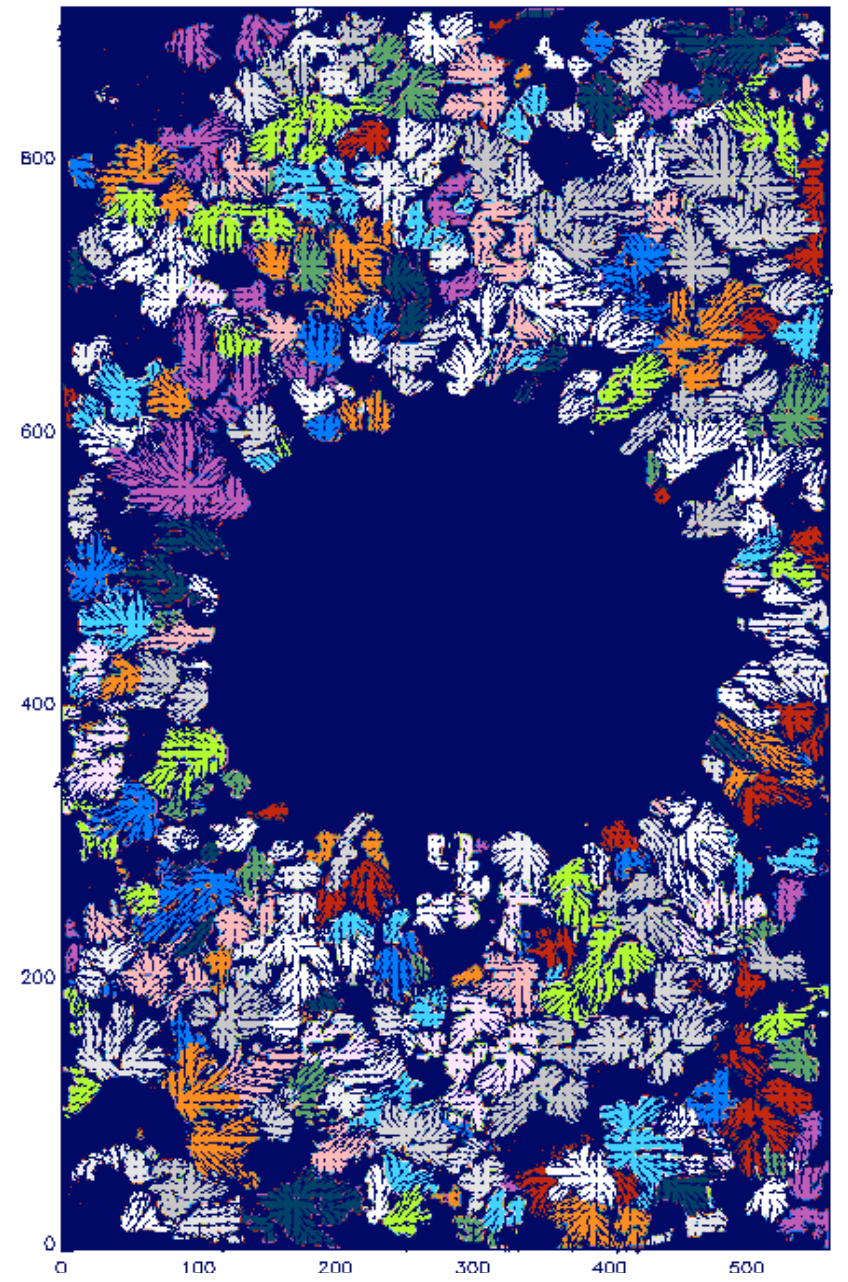

Fig. 5. Areas of families (distinguished by different shades of grey/different colours in the electronic version) superposed on the map of horizontal velocities (LCT, 20-min averaging). The coordinate unit is 0.083 ( 1 pixel).

The area of the family is the total area covered, in the segmented image, by the granules belonging to the family.

Roudier et al. (2003) suggested that the long-lived families are fundamental in structuring the horizontal velocity field in the quiet granulation. The collective action of splitting granules generates diverging flows that, after averaging in time, are detected by LCT in the form of mesogranules. The coherence of families and mesogranules around the sunspot was checked by comparing the LCT velocity maps, computed with a timeaveraging interval of $20 \mathrm{~min}$, with the spatial distribution of families represented by their areas integrated over the same period. An example is shown in Fig. 5. We can see from the figure that the cumulative areas of families and the mesogranules are co-spatial in the whole FOV, including the moat. This suggests that, as in quiet regions, the local divergent motions in the vicinity of sunspots are connected with the long-lived families.

In the moat, local divergent motions of granules are combined with the radial large-scale outflow. This can also be observed in many elongated mesogranules in Fig. 5, where the velocities away from the spot are higher than those directed towards the spot. The family centroids move consistently with

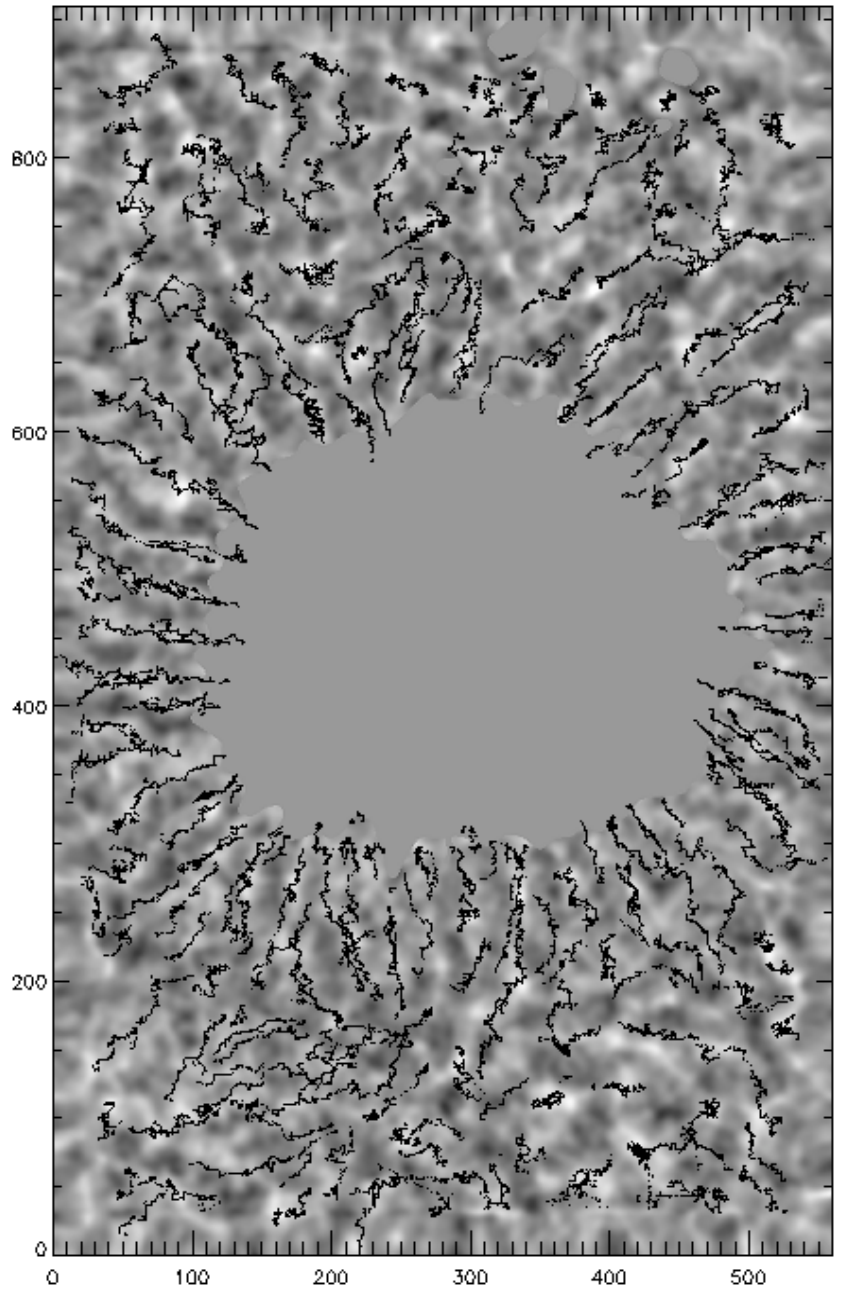

Fig. 6. Trajectories of centroids of the families of granules represented in Fig. 5, superposed on the map of divergence structures (white $=$ positive) averaged over $2 \mathrm{~h}$. The coordinate unit is $0{ }^{\prime} 083$ ( 1 pixel).

this outflow. In Fig. 6 are plotted the trajectories of the centroids of all the long-lived families during the whole 2-h period, superposed on a map of LCT divergence structures, computed with the 2-h time-averaging interval. The trajectories are ordered radially in the moat and are located mostly in the regions of positive divergence. Outside the moat, the trajectories are oriented more randomly, with the exception of the bottom left part of the field, where they are ordered by the flows of a regular supergranule. The average velocities of the centroids (see Table 2, last row) are comparable with the LCT large-scale velocities derived from the motions of divergence structures (Table 2, second row).

\section{3. $G$ band bright points in the moat}

A feature-tracking technique (Sobotka et al. 1997) was applied to the series of segmented images containing GBPs to measure lifetimes, spatial distribution and velocities. In total, 1508 GBPs lasting longer than $2.5 \mathrm{~min}$, with time-averaged diameter $\geq 0$ '. 28 and time-averaged velocity below $4 \mathrm{~km} \mathrm{~s}^{-1}$, were detected in the field of view. The above criteria were set to eliminate noise and improve the reliability of the results. 


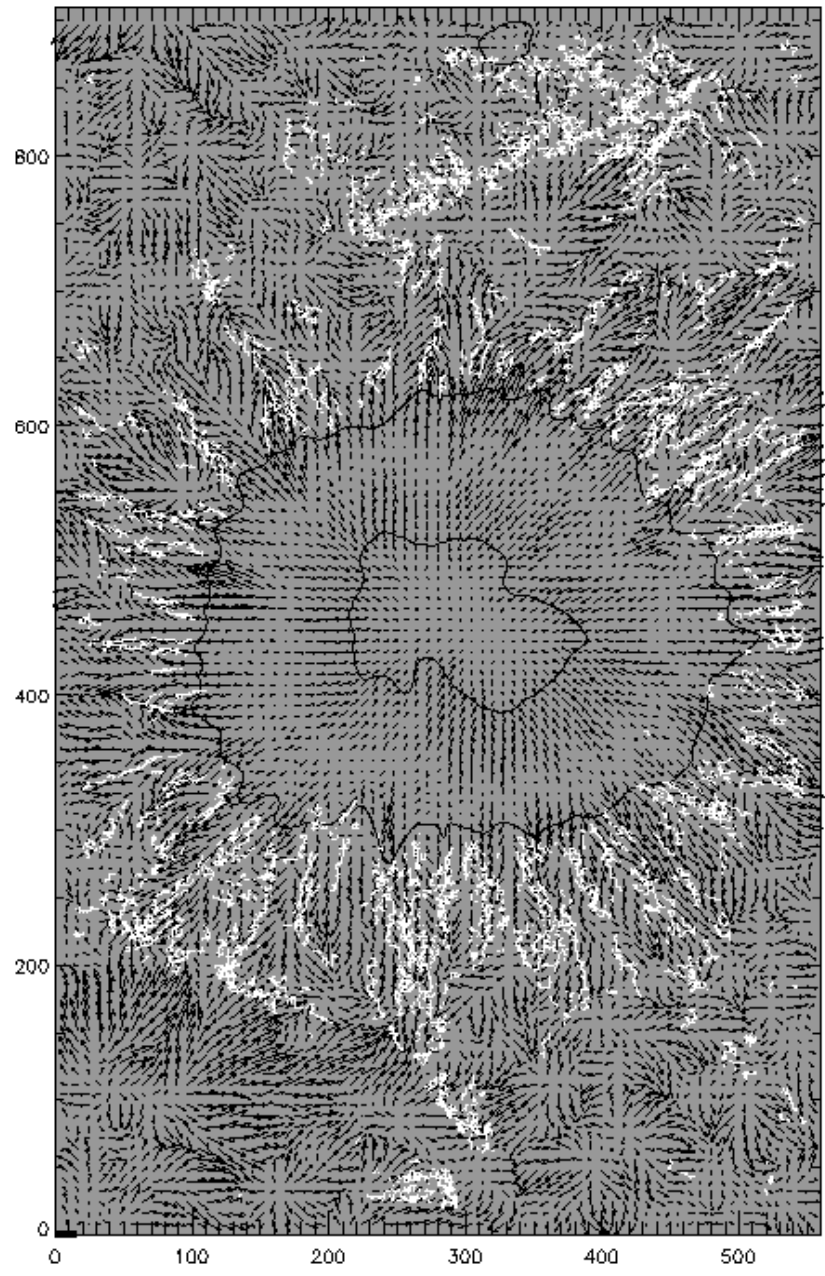

Fig. 7. Positions of GBPs (white) superposed on the map of horizontal velocities (LCT, $2 \mathrm{~h}$ averaging).

About a half of the GBPs (776) were located in the moat as defined in Sect. 3.1. Outside the moat, the GBPs were mostly concentrated around the pores above the sunspot and along the supergranular boundary in the lower part of the field.

The lifetimes of GBPs range from 2.5 to $120 \mathrm{~min}$ (the duration of the series) and the mean is $13 \mathrm{~min}$. The number of GBPs decreases with increasing lifetime. The lifetimes of GBPs inside the moat do not differ from those in the rest of the field. They are in good agreement with those obtained by Muller (1983) in the quiet sun (15 $\mathrm{min})$.

The positions of all 1508 GBPs measured in all frames of the series are plotted in Fig. 7, together with the LCT velocities of granules obtained with the time-averaging interval of $2 \mathrm{~h}$. The LCT velocity pattern in the moat is a combination of diverging flows and the moat's large-scale outflow. The mesogranules appear elongated for this reason. We can see from the figure that the GBPs concentrate to the locations of convergent horizontal motions. Inside the moat, the GBPs are mostly located in "channels" between the diverging flows, or, equivalently, between the areas of families of granules where the repeated granule splitting and expansion take place. It appears that GBPs cannot enter inside the divergent flows.
Table 3. Parameters of outward- and inward-moving GBPs (lifetime > $10 \mathrm{~min}$ ) in the sunspot moat. Standard deviations characterize the width of the distribution of measured values.

\begin{tabular}{lccc}
\hline \hline GBPs & $\begin{array}{c}\text { Lifetime } \\
(\mathrm{min})\end{array}$ & $\begin{array}{c}\text { Displacement } \\
(\operatorname{arcsec})\end{array}$ & $\begin{array}{c}\text { Velocity } \\
\left(\mathrm{km} \mathrm{s}^{-1}\right)\end{array}$ \\
\hline Outward & $28 \pm 23$ & $1.5 \pm 1.0$ & $0.74 \pm 0.40$ \\
Inward & $13 \pm 4$ & $0.7 \pm 0.4$ & $0.69 \pm 0.38$ \\
\hline
\end{tabular}

The time-averaged horizontal velocities of GBPs were calculated from their positions during the lifetime. The clouds of $x$ - and $y$-coordinates versus time were approximated by least-squares linear fits. The slopes of these lines represent the $x$ - and $y$-components of the time-averaged velocities. The measurement accuracy obviously increases with increasing lifetime. For this reason, we used GBPs with lifetimes longer than $10 \mathrm{~min}$. In this case, the velocity is measured with a standard deviation of about $0.05 \mathrm{~km} \mathrm{~s}^{-1}$. We checked the trajectories of GBPs visually to eliminate spurious objects and mistakes in tracking, and obtained a final sample of 249 GBPs in the moat and 194 GBPs in the rest of the field. On average, GBPs inside the moat move faster $\left(0.74 \pm 0.40 \mathrm{~km} \mathrm{~s}^{-1}\right)$ than those outside $\left(0.52 \pm 0.40 \mathrm{~km} \mathrm{~s}^{-1}\right)$. Standard deviations characterize the width of the distribution of measured speeds.

Inside the moat, 238 GBPs move outwards, away from the sunspot, and only 11 GBPs (4.4\%) move towards the spot. Lifetimes, total lengths of displacement and time-averaged velocities of outward- and inward-moving GBPs are summarized in Table 3. We can see that the outward-moving GBPs live longer and travel at longer distances than the inward-moving ones. This fact is probably related to the interaction of GBPs with granules that move mostly away from the sunspot. The inward-moving GBPs are located between exploding granules and the outer penumbral boundary. Their motion is strongly influenced by the expansion of exploding granules.

To check whether GBPs in the moat move "passively", carried along by the same agent as the neighbouring granules, or whether they are driven by a different mechanism, a local comparison of the time-averaged velocity vectors of GBPs and the adjacent granules was done. For this purpose, we used the sample of 249 GBPs with lifetimes longer than $10 \mathrm{~min}$ and the results of the tracking of granules (here the feature-tracking technique was employed also for granules) that lasted longer than $5 \mathrm{~min}$. The granules were represented by the positions of the centroids of their areas given by the number of pixels integrating each segmented granule. To eliminate long-lived small features, possibly identical with GBPs, only granules with timeaveraged diameters larger than 0. '35 were taken into account. The diameter of a granule corresponds to that of a circle with equivalent area. The positions of GBPs and granules were compared each $2.5 \mathrm{~min}$ and the granules within the distance of 1."5 were selected as adjacent to a given GBP. The mean of the timeaveraged velocity vectors of all the granules selected during the GBP's lifetime was then calculated to characterize the average velocity vector of the granular motions near the trajectory of a GBP. Mean velocity magnitude and radial and tangential 
Table 4. Mean velocities of GBPs and neighbouring granules in the moat $\left(\mathrm{km} \mathrm{s}^{-1}\right)$. Standard deviations characterize the width of the distribution of measured values.

\begin{tabular}{lccc}
\hline \hline Velocity & Magnitude & $\begin{array}{c}\text { Radial } \\
\text { component }\end{array}$ & $\begin{array}{c}\text { Tangential } \\
\text { component }\end{array}$ \\
\hline GBPs & $0.74 \pm 0.40$ & $0.62 \pm 0.46$ & $-0.02 \pm 0.33$ \\
Granules & $0.65 \pm 0.29$ & $0.57 \pm 0.32$ & $-0.07 \pm 0.27$ \\
\hline
\end{tabular}

component values of the velocity vectors, calculated with respect to the sunspot centre, are shown in Table 4.

We can see from the table that the average tangential velocity components of GBPs and of adjacent granules are small compared to the velocity magnitudes. This means that motions in the moat have predominantly radial directions. On average, the magnitudes and radial components of velocities are greater for GBPs than for the granules. More information about this effect can be retrieved from Fig. 8, where the histograms of these quantities are plotted. The distribution of GBP velocity magnitudes (solid line) is very similar to that of granules (dotted line) until $1.4 \mathrm{~km} \mathrm{~s}^{-1}$, where a new population of "fast" GBPs begins. Fast moving bright points were described previously by Ryutova et al. (1998), as GBPs in a moat, and by Muller (1983) and Muller et al. (1994), as "white-light bright points" in the quiet photospheric network. This population has no counterpart in the velocities of granules. The same is true for the distribution of radial components, from which we can see that the "fast" GBPs are moving away from the sunspot. Several GBPs also move toward the spot (negative values), coherently with the adjacent granules. In summary, most of the GBPs in the moat move "passively" with velocities comparable to those of the neighbouring granules but there is a small fraction $(6 \%)$ of GBPs that move more rapidly, with speeds higher than $1.4 \mathrm{~km} \mathrm{~s}^{-1}$. The existence of fast GBPs does not necessarily mean that they are special objects intrinsically different to the rest and also does not contradict the hypothesis that a large-scale regular outflow carries in a similar way both the granules and the GBPs. Fast GBPs are probably the result of a reinforcement of their velocities when they are pushed by small fast granular fronts or fragments (see Roudier et al. 1994; Berger \& Title 1996; Berger et al. 1998). This internal granular structure is not detected by our segmentation process.

A detailed inspection of the $G$-band movie reveals the following peculiarities for fast GBPs in the moat.

- Most of them are born at distances not more than 4" from the outer penumbral border.

- Several of them are pushed and accelerated by neighbouring exploding granules.

- Fast GBPs very often move into a well pronounced intergranular space. In some cases their brightness increases at the same time.

- Most fast GBPs are located in the moat in the lower part of the sunspot.

Not only the fast GBPs but also, in general, the moat GBPs show a denser population in the lower and right parts (solar
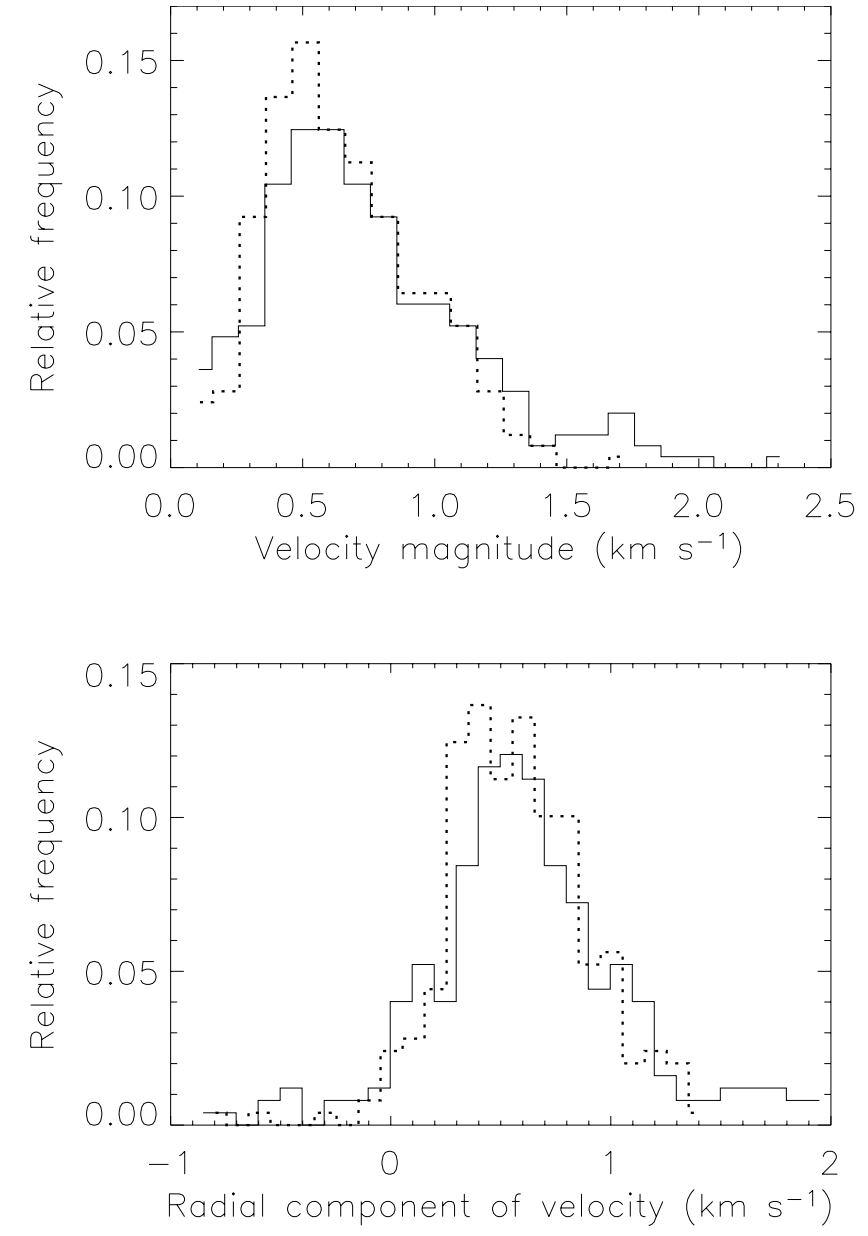

Fig. 8. Histograms of time-averaged velocities of GBPs (solid) and of adjacent granules (dotted) in the moat. Negative radial velocities represent movements toward the sunspot.

limb side of the moat) as shown in Fig. 7. This lack of symmetry could be the result of the inclination of the flux tubes identified as GBPs, in combination with the fact that the sunspot is located out of the disc centre at $\mu=0.93$.

\section{Discussion and conclusions}

Two time series of broad band images of an old regular sunspot, spanning over two hours and taken simultaneously in the $G$-band and white-light were restored for instrumental and atmospheric degradation using the phase diversity technique. The excellent and stable quality of the restored images allowed us to study motions of granules, mesogranules, families of granules and GBPs in the sunspot moat and to measure their timeaveraged velocities. It should be noted that the time-averaged velocities are used to describe the general trends of the motions. These differ from instantaneous velocities, which, for GBPs, can reach $2-3 \mathrm{~km} \mathrm{~s}^{-1}$ and mostly characterize the kinetic energy of the motion. The results can be summarized as follows:

1. Two types of motions of granules are observed in the sunspot moat: a) local divergent motions mostly reflecting the expansion and splitting of granules (mean velocity = $0.64 \mathrm{~km} \mathrm{~s}^{-1}$ ); b) a large-scale regular outflow, which carries 
granules and the centres of divergent motions away from the sunspot (mean velocity $=0.51 \mathrm{~km} \mathrm{~s}^{-1}$ ).

2. Families of repeatedly splitting granules are present in the sunspot moat. The two types of motions $(a, b)$ mentioned above can be identified with the internal evolution of longlived families (a), and with the radially orientated drift of centroids of the families away from the spot (b) - see Fig. 6 . It has also been shown that families of granules and mesogranules are closely related. Thus, an interesting result of this research is that mesogranules and families of granules are advected by the outward flow in the moat. A similar result has been recently found in the quiet Sun, where mesogranules and families also move toward the outer supergranular boundaries (Roudier \& Muller 2004). This gives some support to a supergranular origin of the moat, as first suggested by Sheeley in 1972, on the basis of this similar behaviour. It will be very interesting to find out whether the 3D flows measured by using local helioseismology inversion techniques (Zhao et al. 2001) in decaying sunspots will confirm this hypothesis.

3. Inside the moat, GBPs are mostly located in radially orientated "channels" between the local divergent motions of mesogranular scale and associated families. These motions confine the GBPs within the channels (see Fig. 7).

4. Most GBPs move with the same average speeds as the neighbouring granules. These "passively" moving GBPs are carried along by the same large-scale flows as the granules and families (see Fig. 8). However, some of the GBPs (6\%) move away from the spot more rapidly than the neighbouring granules. This can be interpreted as an extra contribution to their velocities stemming from the interactions with fast moving small granular fronts or fragments. These fast GBPs reach velocities greater than $1.4 \mathrm{~km} \mathrm{~s}^{-1}$ as shown in Fig. 8 .

5. The population of GBPs is denser on the solar limb side of the moat.

6. The lifetimes of GBPs range from 2.5 to $120 \mathrm{~min}$ (the duration of the series). Either inside or outside the moat the lifetimes of GBPs do not differ on average.

Acknowledgements. J.A.B. and I.M. are indebted to M.G. Löfdahl for providing his original PPDS source code in ANA and for his advice in the construction of the IDL code, the basis of the present paper. We are grateful to J. Sánchez Almeida for fruitful discussions during the preparation of this paper. The support provided by R. Kever and G. Hosinsky during the observations is gratefully acknowledged. This paper has been revised for English and style by the Scientific Editorial Service of the IAC. M.S. and J.A.B. thank the Paul Sabbatier University in Toulouse for their stay at the Pic du Midi Observatory. R.M. and M.S. thank the IAC for its hospitality. The Swedish Vacuum Solar Telescope was operated on the island of La Palma by the Royal Swedish Academy of Sciences in the Spanish Observatorio del Roque de los Muchachos of the Instituto de Astrofísica de Canarias. Partial support by the Spanish Ministerio de Ciencia y Tecnología and by FEDER through project AYA2001-1649 is gratefully acknowledged. Part of this work was carried out within the framework of Key Project K2043105 and grant IAA3003404 of the Academy of Sciences of the Czech Republic. This research has been partially supported by the European Community's Human Potential Programme (European Solar Magnetism Network, contract HPRN-CT-2002-00313). The work was also supported by the Observatoire Midi Pyrénées, CNRS,
(UMR 5572) and the Programme National Soleil Terre (PNST). The magnetogram employed in this paper is courtesy of the SOHO/MDI consortium of ESA and NASA.

\section{References}

Berger, T. E., \& Title, A. M. 1996, ApJ, 463, 365

Berger, T. E., Löfdahl, M. G., Shine, R. S., \& Title, A. M. 1998, ApJ, 495, 973

Bonet, J. A., Márquez, I., Muller, R., Sobotka, M., \& Tritschler, A. 2004, A\&A, 423, 737

Gonsalves, R. A. 1982, Opt. Eng., 21, 829

Gonsalves, R. A., \& Childlaw, R. 1979, in Applications of Digital Image Processing III, ed. A. G. Tescher, Proc. Soc. Photo-Opt. Instrum. Eng., 207, 32

Hagenaar, H. J. 2001, ApJ, 555, 448

Harvey, K., \& Harvey, J. 1973, Sol. Phys., 28, 61

Kawaguchi, I. 1980, Sol. Phys., 65, 207

Löfdahl, M. G., \& Scharmer, G. B. 1994, A\&AS, 107, 243

Molowny-Horas, R., \& Yi, Z. 1994, ITA (Oslo) Internal Rep. No. 31

Muller, R. 1983, Sol. Phys., 85, 113

Muller, R., Auffret, H., Roudier, Th., et al. 1992, Nature, 356, 322

Muller, R., \& Ména, B. 1987, Sol. Phys., 112, 295

Muller, R., Roudier, T., Vigneau, J., \& Auffret, H. 1994, A\&A, 283, 232

November, L. J., \& Simon, G. W. 1988, ApJ, 333, 427

Paxman, R. G., Schulz, T. J., \& Fienup, J. R. 1992, J. Opt. Soc. Am., A9, 7, 1072

Paxman, R. G., Seldin, J. H., Löfdahl, M. G., Scharmer, G. B., \& Keller, C. U. 1996, ApJ, 466, 1087

Rieutord, M., Roudier, T., Malherbe, J. M., \& Rincon, F. 2000, A\&A, 357,1063

Roudier, Th., \& Muller, R. 2004, A\&A, 419, 757

Roudier, T., Espagnet, O., Muller, R., \& Vigneau, J. 1994, A\&A, 287, 982

Roudier, T., Bonet, J. A., \& Sobotka, M. 2002, A\&A, 395, 249

Roudier, Th., Lignières, F., Rieutord, M., Brandt, P. N., \& Malherbe, J. M. 2003, A\&A, 409, 299

Ryutova, M., Shine, R. A., Title, A. M., \& Sakai, J. I. 1998, ApJ, 492, 402

Scharmer, G. B., Brown, D. S., Petterson, L., \& Rehn, J. 1985, Appl. Opt., 24, 2558

Scherrer, P. H., Bogart, R. S., Bush, R. I., et al. 1995, Sol. Phys., 162, 129

Schmidt, H. U., Simon, G. W., \& Weiss, N. O. 1985, A\&A, 148, 191

Sheeley, N. R. 1969, Sol. Phys., 9, 347

Sheeley, N. R. 1972, Sol. Phys., 25, 98

Sheeley, N. R., \& Bhatnagar, A. 1971, Sol. Phys., 19, 338

Shine, R. A., Title, A. M., Tarbell, T. D., \& Topka, K. P. 1987, Science, 238, 1203

Sobotka, M., Brandt, P. N., \& Simon, G. W. 1997, A\&A, 328, 682

Sobotka, M., Vázquez, M., Bonet, J. A., Hanslmeier, A., \& Hirzberger, J. 1999, ApJ, 511, 436

Strous, L. 1994, Dynamics in Solar Active Regions: Patterns in Magnetic-flux Emergence, Ph.D. Thesis, University of Utrecht

Thomas, J. H., Weiss, N. O., Tobias, S. M., \& Brummel, N. H. 2002, Nature, 420, 390

Vrabec, D. 1971, in Solar Magnetic Fields, ed. R. Howard (Dordrecht: Reidel), IAU Symp., 43, 329

Wallenhorst, S. G., \& Topka, K. P. 1982, Sol. Phys., 81, 33

Zhao, J., Kosovichev, A. G., \& Duvall, Jr., T. L. 2001, ApJ, 557, 384 